\title{
Nanoarchitecture Effects on Persistent Room Temperature Photoconductivity and Thermal Conductivity in Ceramic Semiconductors: Mesoporous, Yolk-Shell, and Hollow ZnO Spheres
}

\author{
Stefan Dilger, ${ }^{\dagger}$ Martin Wessig, ${ }^{\dagger}$ Markus R. Wagner, ${ }^{\ddagger}$ Juan S. Reparaz, ${ }^{\ddagger}$ Clivia M. Sotomayor Torres, ${ }^{\ddagger}$, \\ Liang Qijun, ${ }^{\dagger}$ Thomas Dekorsy, ${ }^{\dagger}$ and Sebastian Polarz ${ }^{\dagger}$ \\ †University of Konstanz Universitaetsstrasse 10, 78457 Konstanz, Germany \\ *Catalan Institute of Nanoscience and Nanotechnology ICN2, Campus UAB, Edifici ICN2, 08193 Bellaterra (Barcelona), Spain \\ ${ }^{\S}$ Catalan Institution for Research and Advanced Studies ICREA, 08010 Barcelona, Spain
}

Supporting Information

ABSTRACT: Whereas size effects have been investigated extensively and are largely understood, it is significantly more challenging to elucidate how functional properties of semiconductors can be altered and ultimately be improved by a hierarchical nanoarchitecture. For semiconductor applications, such as in photovoltaics or photocatalysis, it is of great importance to learn how to avoid the recombination of photogenerated charge carriers and how to enhance their lifetime. A gas phase synthesis method is explored, which enables the generation of spherical zinc oxide nanostructures with compact, mesoporous, a special type of core-shell, so called yolk-shell, or hollow character. The particles with hollow character exhibit an extraordinarily long persistence of photogenerated charge carriers. It is demonstrated that the presence of the $\mathrm{ZnO}$ shell and its special orientation with respect to the polar character of the wurtzite lattice represent deciding factors. After photoexcitation, electrons and holes migrate to opposite sides of the interfaces, where they are

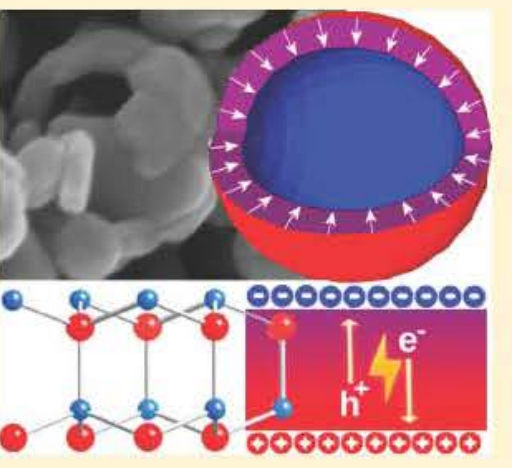
stabilized. Moreover, photoluminescence thermometry was used to determine the thermal conductivity of the samples, which is lowered by a factor of $\sim 100$ compared with bulk $\mathrm{ZnO}$. The thermal conductivity of this type of nanostructure is found to be only 10 times larger than that of air, and this points toward potential applications as thermoelectrics.

\section{INTRODUCTION}

Ceramic semiconductors continue to attract strong interest because they play a major role in many contemporary applications. For example, III/V compounds like GaN are currently revolutionizing lighting technologies (LEDs) ${ }^{1}$ and II/ VI compounds are applied for UV protection, ${ }^{2}$ in photovoltaic devices, $^{3,4}$ or as photocatalysts, for example, for the catalytic splitting of water or waste treatment. ${ }^{s-7}$ The performance of many of these devices depends critically on the ability of the semiconductors to generate excitonic charge carriers (elec tron-hole pairs) when irradiated with light, as well as on the persistence of those photogenerated charge carriers (PGCCs). Thus, it is of prime importance to understand how the lifetime of these PGCCs is affected by the specific properties of the materials and how it can be controlled. ${ }^{8}$ The latter still remains a substantial challenge since the actual persistence of PGCCs depends strongly on numerous factors such as the coverage with stabilizers or other compounds, the density of intrinsic and extrinsic defects and, last but not least, the hierarchical construction of the material from the nanometer to the macroscopic scale. The structural motifs themselves evolve to a large extent from kinetic factors and are determined by the interplay of many parameters, which differ during the material syntheses. Unfortunately, it is very cumbersome to elucidate systematic coherences, when every material requires a different synthesis strategy. ${ }^{9-12}$ Nevertheless, the idea is highly tempting that the persistence of PGCCs could be controlled via the synthesis of materials with precisely determined nanostructure. Whereas this goal is currently out of reach, the progress reported in this work represents one step into this direction. We show how the lifetime of PGCCs is affected in $\mathrm{ZnO}$ nanostructures that differ systematically in their architecture. Comprehensive analytical investigations are performed in order to clarify which structural motif is responsible for persistence of PGCCs.

$\mathrm{ZnO}$ is an ideal material for the mentioned purpose, not only because it is a prominent, direct wide band gap semi conductor ${ }^{13}$ but also because of its crystal structure (wurtzite; $\mathrm{P}_{3} \mathrm{mc}$ ), which promotes strong electric fields between its polar faces. A multitude of different $\mathrm{ZnO}$ nanostructures have been reported in literature. ${ }^{14}$ One morphology that has gained increasing attention in recent years is spherical particles in the 
size regime $>50 \mathrm{~nm}^{15-23}$ Nevertheless, examples for spherical $\mathrm{ZnO}$ particles with a more complex substructure are rare. Yan and co workers prepared spherical $\mathrm{ZnO}$ nanostructures varying from flower like to urchin like morphology, ${ }^{15}$ and recently Zhang and co workers obtained multishell hollow $\mathrm{ZnO}$ spheres. ${ }^{24}$ Another highly unusual structure is the so called nanorattle or yolk/shell particle morphology. ${ }^{25,26}$ An impressive paper was presented by $\mathrm{Oh}$ and co workers. ${ }^{27}$ The authors were able to synthesize yolk/shell oxide spheres with adjustable ratios of two different oxides starting from coordination polymer spheres containing two different metals. The yolk/ shell morphology in particular has many potential applications, for example, in battery and capacitor technologies and for drug release, gas sensing, and confined nanocatalysis. ${ }^{25,28-34}$

However, the latter preparation routes are disadvantageous for our aim since they all differ in key synthesis parameters, which might affect the defect structure in an unpredictable way. Furthermore, these materials might still contain residues (e.g., organic substances such as surfactants or solvents), and crystallinity is often poor in sol-gel routes. ${ }^{35,36}$ An alternative preparation approach needs to be developed for which all desired nanostructures can be obtained using only one method and preferably with changes of only few synthesis parameters. Under these conditions, it can be expected that intrinsic defects would be homogeneous for the entire set of materials and their influence on electronic properties could much more easily be identified and isolated.

\section{EXPERIMENTAL SECTION}

Materials Preparation. A series of quartz tubes was used for the gas phase synthesis. Tube ovens from Nabertherm were used to generate zones of different temperature and mass flow controllers from Bronkhorst (model EL FLOW Select) were used to adjust the gas flow $\left(\mathrm{N}_{2}\right.$ and $\left.\mathrm{O}_{2}\right)$ through the tube reactor. Flow rates of $f_{\text {nitrogen }}=0.6 \mathrm{~L} /$ min and $f_{\text {oxygen }}=0.15 \mathrm{~L} / \mathrm{min}$ were chosen for all syntheses. The liquid precursor $\left[\mathrm{MeZnOCH}_{2} \mathrm{CH}_{2} \mathrm{OMe}\right]_{4}$ was injected into the $70 \mathrm{~cm}$ evaporation zone (EZ; Supporting Information, Figure S 1, module I) of the reactor with a syringe pump from KD Scientific model KS200. An injection rate of $100 \mu \mathrm{L} / \mathrm{h}$ and an evaporation temperature of 110 ${ }^{\circ} \mathrm{C}$ was chosen for all syntheses. This allows the precursor to enter the $200 \mathrm{~cm}$ long particle formation zone (PFZ, module II + III in Supporting Information, Figure S 1) in gaseous form. The precursor concentration is determined by the injection rate and the volume of the added gases. In addition the concentration could be determined experimentally from the integration of the particle distribution function measured with an SMPS setup from TSI (see Supporting Information, Figure S 1 , and Characterization Methods section). The flow rate of the gases together with the length of the $2.7 \mathrm{~cm}$ diameter quartz tubes determines the residence time of the aerosol in the different zones of the reactor. A custom built thermophoresis unit was used for the separation of the particles from the aerosol. Amorphous spheres were synthesized with a reactor length of $130 \mathrm{~cm}$ in module II and $70 \mathrm{~cm}$ in module III (see Supporting Information, Figure S 1) The decomposition temperature was set to $120^{\circ} \mathrm{C}$ in both module II and III. To obtain crystalline spheres, the temperature in module III was raised. For $400{ }^{\circ} \mathrm{C}$, dense spheres are obtained. From 600 to $900{ }^{\circ} \mathrm{C}$, yolk/shell spheres can be formed, and hollow spheres form from 700 to $1200{ }^{\circ} \mathrm{C}$. For the synthesis of the hollow spheres, the residence time in module III was shortened to $2 \mathrm{~s}$ by introducing an additional stream of $f_{\text {nitrogen }}=1.32 \mathrm{~L} / \mathrm{min}$ just before the module. Hollow $\mathrm{ZnO}$ spheres with crystallite sizes $\sim 10 \mathrm{~nm}$ can additionally be obtained with temperatures of $400-700{ }^{\circ} \mathrm{C}$ in module III if the oxygen stream is enriched with 2 equiv of additional alcohol residue $\mathrm{MeOCH}_{2} \mathrm{CH}_{2} \mathrm{OH}$ per zinc before entering module II. To obtain porous $\mathrm{ZnO}$ spheres, the amorphous intermediate synthesized at $120^{\circ} \mathrm{C}$ reactor temperature was aged in air at 40,60 , and $80^{\circ} \mathrm{C}$ for 3 days.
Sensor Preparation. Four milligrams of the $\mathrm{ZnO}$ spheres was dispersed in $5 \mathrm{~mL}$ of deionized water by ultrasonication. One drop of the suspension was added on a $3 \times 3 \mathrm{~mm}^{2}$ sensor grid by Umweltsensortechnik and allowed to dry. The sensors were sintered at $200{ }^{\circ} \mathrm{C}$ under a $92 \%$ oxygen, $8 \%$ ozone atmosphere for $3 \mathrm{~h}$. Ozone was produced by a Pacific ozone gas generator LAB11 with a corona voltage of $5 \mathrm{~V}$. Photoconductivity measurements were carried out in a custom measurement chamber. ${ }^{37}$ Conductivity was measured with a Zahner IM 6. For UV illumination, a lab grade UV lamp with a power density of $91.2 \mathrm{~mW} / \mathrm{cm}^{2}$ and a peak intensity of $366 \mathrm{~nm}$ was used.

Characterization Methods. The aerosol was analyzed using equipment from TSI (electrostatic classifier 3080; condensation particle counter 3775; differential mobility analyzer 3081 and 3085). FT IR spectra were measured on a PerkinElmer Spectrum 100 spectrometer. PXRD data were acquired on a Bruker D8 Advance. SEM measurements were performed on a Zeiss CrossBeam 1540XB. Conventional TEM measurements were performed on a Zeiss Libra 120; high resolution TEM measurements were performed on a Jeol JEM2200FS. $\mathrm{N}_{2}$ physisorption measurements were acquired on a Micromeritics Tristar. TGA measurements were acquired on equip ment from Netzsch (STA 429). Continuous wave (cw) ESR measurements were performed on an $\mathrm{X}$ band Miniscope spectrometer (MS200, Magnettech $\mathrm{GmbH}$ ) equipped with a variable temperature unit (temperature controller TC H02, Magnettech GmbH). The time domain terahertz spectroscopy was performed in a standard terahertz transmission geometry based on the ASOPS system (Gigajet TWIN, Gigaoptics/Laserquantum $\mathrm{GmbH}$ ). The core element of the ASOPS system are two mode locked Ti:sapphire oscillators with repetition rate of about $1 \mathrm{GHz}$ and a stabilized small repetition rate offset of 2 $\mathrm{kHz}$. The center wavelength and duration of the two lasers are about $800 \mathrm{~nm}$ and $50 \mathrm{fs}$, respectively. The time domain spectra of the reference and the sample were obtained by measuring the terahertz transmission of the bare glass and the thin $\mathrm{ZnO}$ film on the glass, respectively. The frequency domain spectra were obtained by performing Fourier transform to the time domain spectra. Then, the terahertz transmission through the thin $\mathrm{ZnO}$ film was calculated by division of the sample spectrum by the reference spectrum. Raman measurements were performed using a HORIBA T64000 Raman setup with the $514.5 \mathrm{~nm}$ laser line of an $\mathrm{Ar}$ ion laser as excitation source. The laser was focused by a $100 \times(\mathrm{NA}=0.9)$ microscope objective to a laser spot of about $0.5 \mu \mathrm{m}$ in diameter. The laser power was set to 0.5 $\mathrm{mW}$ in order to avoid heating of the samples. Raman spectra were recorded by a charge coupled device (CCD) in backscattering geometry. Low temperature luminescence spectra were measured using an oxford He microcryostat with variable temperature between 4 and $500 \mathrm{~K}$. The thermal conductivity of the samples was determined using a variation of the effective medium approximation as described in refs $38-40$, which we are referring to as photoluminescence thermometry. In these works, the thermal conductivity of a semi infinite medium $\left(k_{\text {eff }}\right)$ is obtained through the temperature rise $\left(T_{\text {rise }}\right)$ resulting from focusing a monochromatic Gaussian light source (laser source) with diameter $d$ onto the surface of the sample and considering an absorbed power $P$ with $k_{\text {eff }}=2 P /\left(\pi d T_{\text {rise }}\right)$. The diameter of the focused laser spot, $d=740 \mathrm{~nm}$, was obtained from the diameter of the first Airy disk for a $40 \times \mathrm{UV}$ enhanced objective with a numerical aperture NA $=0.5$ and an excitation wavelength of $350.7 \mathrm{~nm}$ ( $\mathrm{Kr}$ ion gas laser). The absorbed power was determined by the incident power times the absorption coefficient $A=0.8$ for the incident wavelength as extracted from ref 41 . The temperature rise was measured by the spectral position of the near band edge emission for different absorbed powers (Supporting Information, Figure S 10). With use of the temperature dependence of the PL emission based on a Bose-Einstein model from ref 42 , the spectral position of the PL maximum is translated to a temperature rise due to the local heating of the sample by the laser. Finally, with the above given equation, the thermal conductivity of the medium is computed from the measured quantities. 


\section{RESULTS AND DISCUSSION}

Materials Synthesis and Structure. We utilize the advantages of gas phase processing concerning solvent and surfactant free conditions s $^{37,43-45}$ and combine this approach with a volatile organometallic precursor suitable for $\mathrm{ZnO}$ formation: $\left[\mathrm{MeZnOCH}_{2} \mathrm{CH}_{2} \mathrm{OMe}\right]_{4}{ }^{46-50}$ An important, pre liminary result is that the generation of $\mathrm{ZnO}$ from this precursor proceeds via an amorphous, polymeric transition state, $\left[\mathrm{HOZnO}\left(\mathrm{HOCH}_{2} \mathrm{CH}_{2} \mathrm{OMe}\right)\right]_{n}{ }^{51-53}$ The amorphous character of this phase can be stabilized at low temperatures for a short time. Due to collision of viscous droplets in the gas phase, followed by coagulation, a growth process of amorphous particles in the size range $30-500 \mathrm{~nm}$ could be initiated in the aerosol state (Figure 1a; see also Supporting Information,

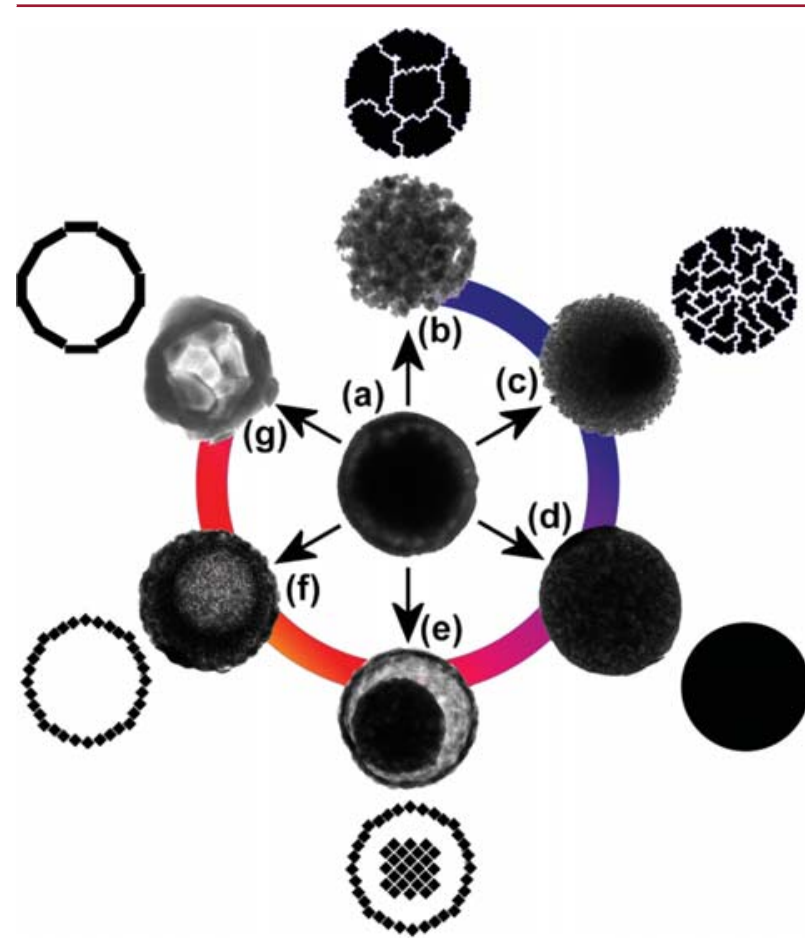

Figure 1. Structural evolution of different spherical $\mathrm{ZnO}$ nanomateri als (TEM data and schematic cross section) depending on different temperature conditions applied to the amorphous intermediate phase (a). Porous $\mathrm{ZnO}$ particles prepared at $\mathrm{T}=40{ }^{\circ} \mathrm{C}$ (b) and $80^{\circ} \mathrm{C}$ (c). (d) Solid spheres prepared at $T=400{ }^{\circ} \mathrm{C}$; (e) yolk-shell structure prepared at $T=600^{\circ} \mathrm{C}$; (f, g) hollow spheres with different crystallite size prepared at $T=700{ }^{\circ} \mathrm{C}$ and $T=1200{ }^{\circ} \mathrm{C}$. Additional EM data is shown for each system in the Supporting Information.

Figure $S$ 1). The amorphous character was proven by electron diffraction (ED) and powder $\mathrm{X}$ ray diffraction (PXRD) (data given in Supporting Information, Figure S 2). The occurrence of the spherical particles with amorphous character is a deciding bifurcation point, which enables the generation of an entire collection of $\mathrm{ZnO}$ nanomaterials (summarized in Figure 1) using one single preparation method.

For this purpose, the aerosol is led into a zone of different temperature directly after generation of the amorphous particles (see Supporting Information, Figure S 1). There, the loss of the volatile organic compounds, solidification, and $\mathrm{ZnO}$ crystallization takes place (see also Supporting Information, Figure S 3). The kinetics of the latter processes is decisively influenced by temperature. One can differentiate between two regimes. Treatment of the amorphous spheres at low temperatures $\left(T=40-100{ }^{\circ} \mathrm{C}\right)$ leads to particles with different degrees of porosity. The pore size of these materials is in the mesoporous range and is inversely proportional to temperature (Figure 1b,c; Supporting Information, Figure S 4). Scanning electron microscopy (SEM) and nitrogen physisorption data are shown for the material prepared at $T=80{ }^{\circ} \mathrm{C}$ as an exemplary case in Figure 2. The formation of the mesoporous

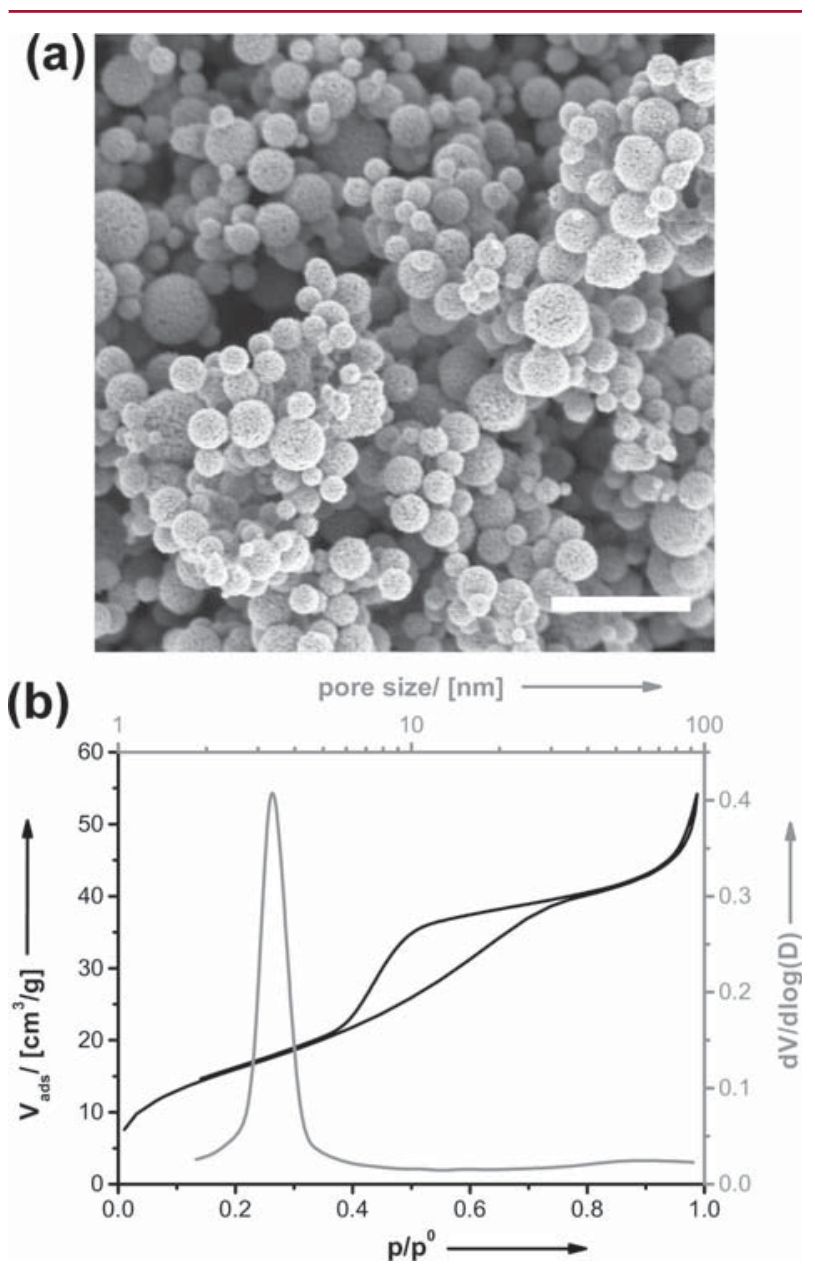

Figure 2. (a) SEM micrograph of mesoporous $\mathrm{ZnO}$ spheres prepared at $T=80^{\circ} \mathrm{C}$. Scale bar $\cong 1 \mu \mathrm{m}$. (b) Nitrogen physisorption data and $\mathrm{BJH}$ pore size distribution function.

$\mathrm{ZnO}$ materials is remarkable, because porous solids with similarly narrow pore size distribution can usually only be obtained by using organic template structures. ${ }^{49}$ In contrast, no template was used in the method described here.

The second regime is realized at higher temperatures (400$1200{ }^{\circ} \mathrm{C}$ ). At $400{ }^{\circ} \mathrm{C}$, one is able to transform the amorphous particles into dense $\mathrm{ZnO}$ spheres (Figure 1d). SEM and TEM investigations as well as $\mathrm{N}_{2}$ physisorption (given in Supporting Information, Figure S 5) indicate smooth surfaces without any porosity.

Higher temperatures during the gas phase process induce a temperature gradient, which leads to a rapid crystallization at the outer shell with a subsequent mass transport from the inner core to the newly formed hull (see Scheme 1). This effect 
resulting in the formation of $3 \mathrm{D}$ defects (pores and cavities) has rarely been observed also for other nanomaterial systems and is known as the Kirkendall effect. ${ }^{24,26,54}$

Scheme 1. Schematic Illustration of the Temperature Gradient Acting on the Amorphous Particles in the Gas Phase ( $c \cong$ Center of the Particle; $s \cong$ Surface of the Particle) and Its Effect on the Morphology of the Resulting ZnO Particles

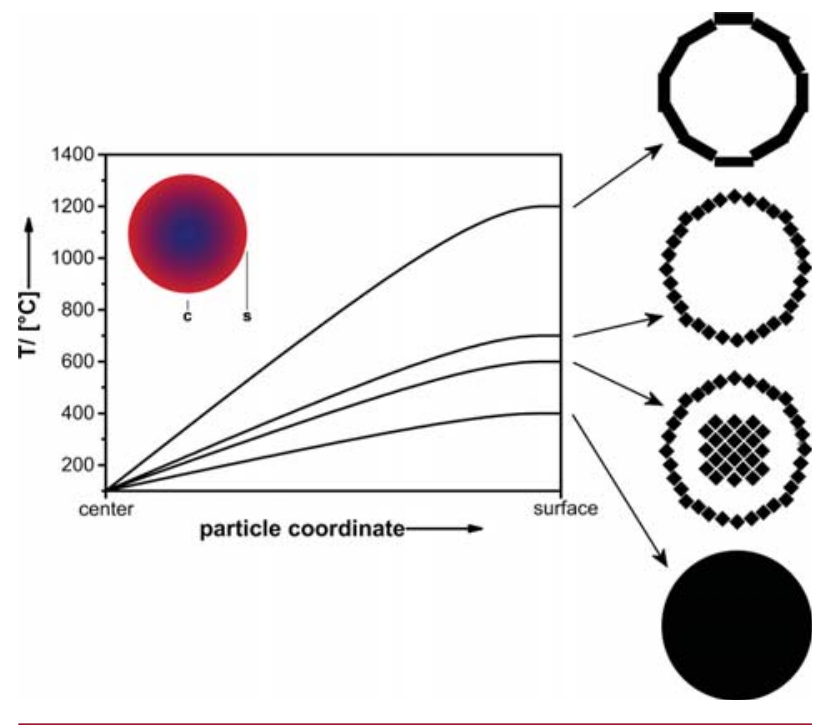

At $600{ }^{\circ} \mathrm{C}$, we could clearly identify a yolk-shell morphology (Figure 1e; Supporting Information, Figure S 6). Due to the rapid crystallization rates, the surface becomes rigid, whereas the core of the particles is still amorphous and soft. With increasing temperature, the core shrinks, which is caused by the evaporation of volatile constituents of the precursors. Eventually, the core tears off from the shell.

At even higher temperatures all internal matter migrates to the surfaces resulting in hollow $\mathrm{ZnO}$ spheres. The structure of the material obtained at $1200{ }^{\circ} \mathrm{C}$ is remarkable in many respects. Such high temperatures induce an expected growth of the $\mathrm{ZnO}$ crystallites via sintering as demonstrated by the peak narrowing in PXRD (shown in Supporting Information, Figure $\left.\mathrm{S} 7 ; D_{600^{\circ} \mathrm{C}}=13.5 \mathrm{~nm} ; D_{1200^{\circ} \mathrm{C}}=49.5 \mathrm{~nm}\right)$ and their evaluation using full profile analysis. However, SEM (given in Supporting Information, Figure S 8) and TEM (Figure 3a) images clearly show that the spherical, hollow nanostructure is still present with the shell of the hollow particles being composed of several crystalline platelets.

The frequently observed hexagonal shapes and $120^{\circ}$ and $60^{\circ}$ angles in the images are a first indication that the external surface might correspond to the [002] lattice plane of the wurtzite structure. Lattice fringes of the particle shell could be visualized by high resolution TEM at the periphery of the particles as shown in Figure 3a,b. The fast Fourier trans formation (FFT) pattern taken from these positions with atomic resolution are shown in Figure $3 \mathrm{e}$. The results are in agreement with the expected $\mathrm{ED}$ pattern for $\mathrm{ZnO}$ crystals with an orientation along the [100] and [110] lattice planes of the wurtzite structure (Figure $3 \mathrm{f}$ ). The distances determined (2.83 $\AA$, respectively $1.80 \AA$ ) fit well to the expected values for the [100], respectively [110] planes. Consequently, the surface of the particles is the plane perpendicular to [100] and [110], which is the [002] plane (surface normal to the $c$ axis). Furthermore, micro Raman spectra were recorded and are shown in Figure 4a. The spectrum of the hollow $\mathrm{ZnO}$ spheres is
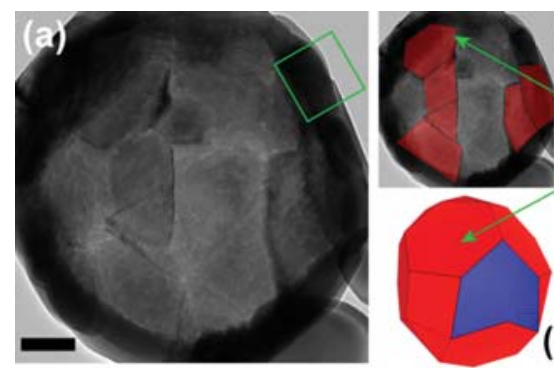

(b)
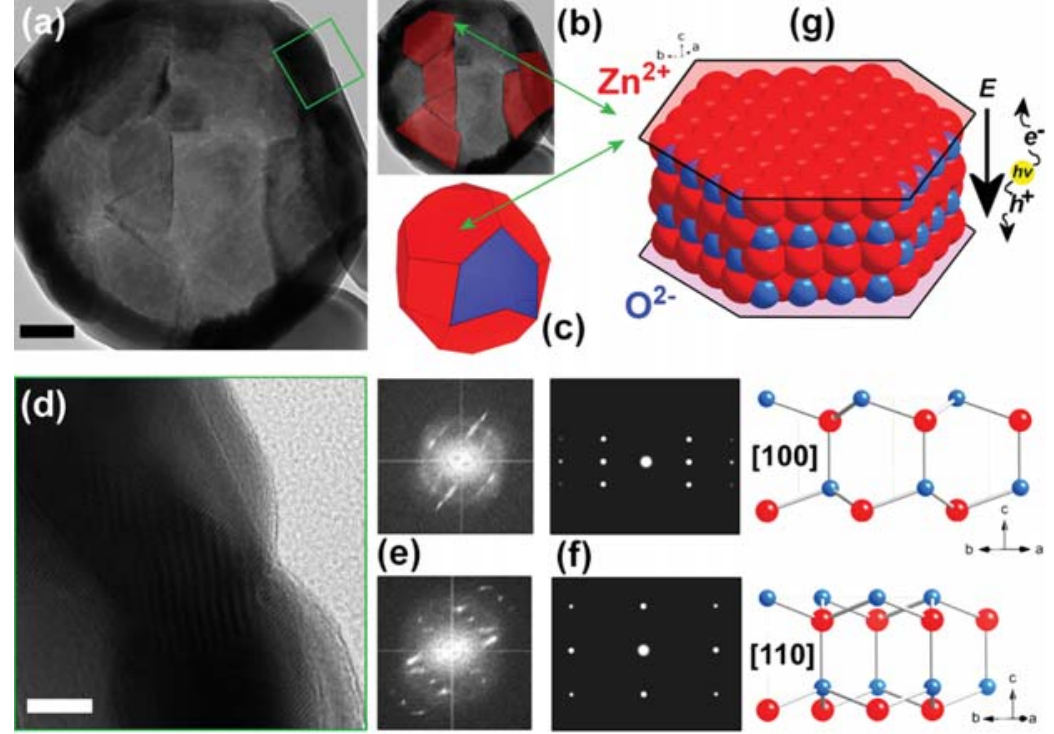

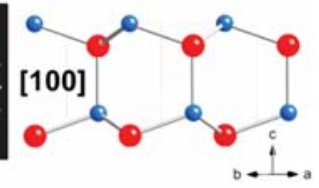

(f)
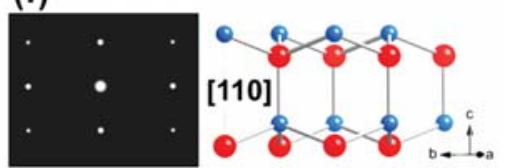

Figure 3. (a) TEM image of a single hollow $\mathrm{ZnO}$ nanosphere. Scale bar $\cong 50 \mathrm{~nm}$. (b, c) Crystal shapes occurring on the surface of the hollow particles (red) and a schematic representation of one hollow particle containing a single open face (see also Figure S 8, Supporting Information). (d) HRTEM image taken from the periphery of the hollow particle. Scale bar $\cong 10 \mathrm{~nm}$. Larger images are shown in Figure $S$ 8, Supporting Information. (e) Fast Fourier transformation (FFT) taken from the two outer crystallite positions in image d (top region and bottom region). (f) Lattice representation and correlating $\mathrm{ED}$ of two planes perpendicular to the $c$ axis (the polar axis). (g) Resulting model for the arrangement of polar $\mathrm{ZnO}$ crystallites in the hollow particles. The polarity of the particles creates an electric field $(E),{ }^{57}$ which acts differently on photogenerated $(h \nu)$ charge carriers $\left(\mathrm{h}^{+}, \mathrm{e}^{-}\right)$. 


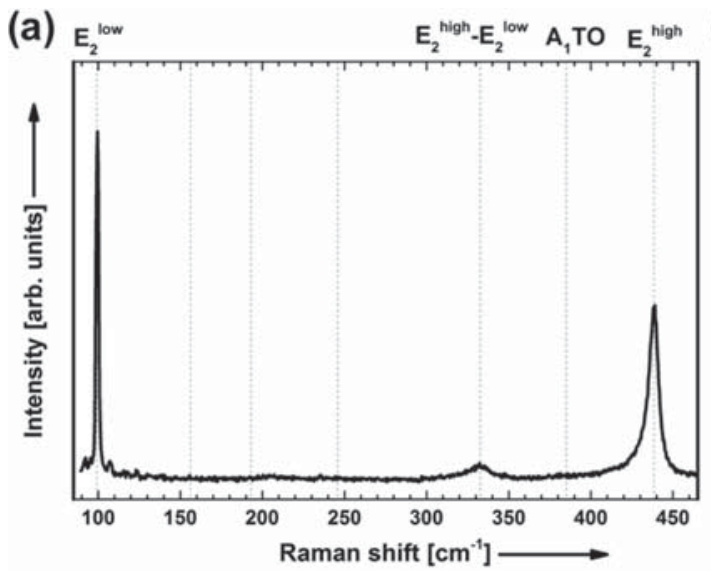

(b)

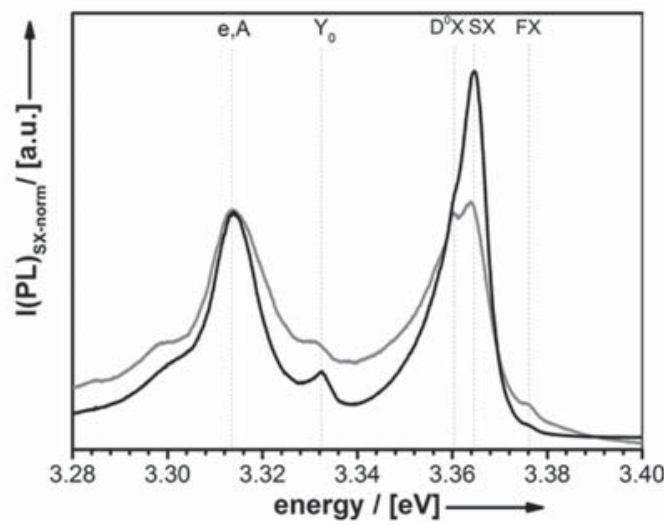

Figure 4. (a) Micro Raman spectrum of hollow $\mathrm{ZnO}$ nanospheres at room temperature. (b) Low temperature PL spectra of hollow $\mathrm{ZnO}$ spheres (black graph) and compact $\mathrm{ZnO}$ spheres (gray graph).

dominated by the characteristic vibrational modes $\mathrm{E}_{2}^{\text {low }}$ at $\sim 98$ $\mathrm{cm}^{-1}$ and $\mathrm{E}_{2}^{\text {high }}$ at $\sim 438 \mathrm{~cm}^{-1}$ of the $\mathrm{ZnO}$ lattice. ${ }^{55}$ In addition, the second order mode $\mathrm{E}_{2}^{\text {high }}-\mathrm{E}_{2}^{\text {low }}$ at $\sim 332 \mathrm{~cm}^{-1}$ is visible. It is noteworthy that both TO modes, the $\mathrm{A}_{1}(\mathrm{TO})$ at $\sim 377 \mathrm{~cm}^{-1}$ and the $\mathrm{E}_{1}(\mathrm{TO})$ at $\sim 411 \mathrm{~cm}^{-1}$, are absent in the spectra. According to the Raman scattering tensors, these modes are only visible in the $x(\ldots) x$ geometry but forbidden for the $z(\ldots) z$ configuration. $^{56}$

In contrast, polycrystalline $\mathrm{ZnO}$ or nanoparticles with random orientation are known to exhibit a strong signal of the TO modes. Consequently, the absence of the TO modes indicates that the incident and the scattered light is mainly oriented parallel to the $c$ axis. Taking the spherical symmetry of the nanospheres into account, the Raman spectra therefore confirm the model of the [002] polar faces oriented tangential to the sphere.

Photophysical Properties. The yolk-shell and the hollow $\mathrm{ZnO}$ spheres were used to assemble photodetectors in analogy to procedures reported in literature (see also Experimental Section for details). ${ }^{58-60}$ The photocurrent was measured as a function of time after illumination using an UV lamp $\left(\lambda_{\max }=\right.$ $366 \mathrm{~nm}$ ) with an excitation density of $91.2 \mathrm{~mW} / \mathrm{cm}^{2}$ for 15 min. In the case of bulk $\mathrm{ZnO}$, the photocurrent decays rapidly due to recombination of the PGCCs as shown in Figure 5a.

The time needed for the current to drop down to $10 \%$ of its maximum value $\left(T_{0.1}\right)$ is only $20 \mathrm{~s}$. In contrast to that, it becomes evident that the different nanostructures have a significant effect on the lifetime of the PGCCs. The $T_{0.1}$ times in these materials are orders of magnitude larger reaching values of $521 \mathrm{~s}$ for the yolk-shell spheres and $1150 \mathrm{~s}$ in case of the hollow $\mathrm{ZnO}$ spheres (Figure 5a, sample iii). Even $2 \mathrm{~h}$ (7200 s) after illumination, one can clearly detect an enhanced conductivity of the samples. We suggest that the hollow nature of the particles and not the preparation method is responsible for this effect as supported by the fact that the porous $\mathrm{ZnO}$ particles (Figure $1 \mathrm{~b}, \mathrm{c}$ ) behave similarly to bulk $\mathrm{ZnO}$ with short relaxation periods of $T_{0.1}=60 \mathrm{~s}$. (see Supporting Information, Figure $S$ 9). Another powerful technique for the investigation of charge carriers in semiconductors is electron paramagnetic resonance (EPR) spectroscopy ${ }^{48,61-63}$ In the case of bulk $\mathrm{ZnO}$, a weak EPR signal is observed with a $g$ factor of 1.96 during UV radiation, which vanishes quickly after the illumination has been turned off (Figure 5b).
The same signal is found for the spherical nanoparticles presented in the current study; however, the intensity is much stronger and its persistence is much larger (Figure 5c). Again, it is obvious that the morphology of the samples is a deciding factor. The persistence of the PGCCs detected using EPR increases significantly for an increasing amount of the $\mathrm{ZnO}$ shell, starting from compact spheres over yolk-shell to hollow spheres. Specifically for the hollow spheres, there is no discernible intensity loss visible during $8000 \mathrm{~s}$. Stable room temperature photoconductivity has very seldom been reported for $\mathrm{ZnO},{ }^{64-68}$ and the decay times reported here belong to the longest ever found.

Whereas photoconductivity measurements and EPR spec troscopy are suitable techniques for investigating the persistence of PGCCs on long time scales, other methods have to be applied for the investigation of shorter time scales. The picosecond time domain can be probed using time resolved photoluminescence spectroscopy (TRPL). Figure 6a shows the decay of the low temperature band acceptor (e, A) transition for the compact and hollow nanospheres (see also the PL spectrum in Figure 4b). The observed biexponential decay with time constants of $340 \mathrm{ps}$ and $1.15 \mathrm{~ns}$ is in excellent agreement with previously reported lifetimes in $\mathrm{Li}$ doped $\mathrm{ZnO}$ nanocrystals ${ }^{69}$ with no significant difference between solid spheres and hollow spheres. These findings are corroborated by time domain terahertz transmission experiments. Time domain terahertz spectroscopy (TDTS) allows the determination of the dynamic conductivity of nanoparticles without the need for electrical contacts. ${ }^{70-72}$ The time domain terahertz spectrosco py system is based on asynchronous optical sampling (ASOPS). ASOPS relies on two femtosecond lasers for impulsive generation and time domain detection of broadband terahertz pulses. Details of the experimental setup can be found elsewhere. ${ }^{73}$ The yolk-shell particles deposited with $\sim 3 \mu \mathrm{m}$ thickness on a glass substrate show a few percent absorption in the terahertz frequency range. Figure $6 \mathrm{~b}$ shows the terahertz transmission spectrum of the microspheres with and without UV exposure, as well as after $2400 \mathrm{~s}$ after UV exposure. The data are obtained from Fourier transforms of the time domain data and division through a reference spectrum of the glass substrate only. Values with transmission larger than 1.0 stem from decreased reflectivity. The transmission spectra show in all cases oscillatory components, which are not yet assigned. However, compared with the nonirradiated sample, the 


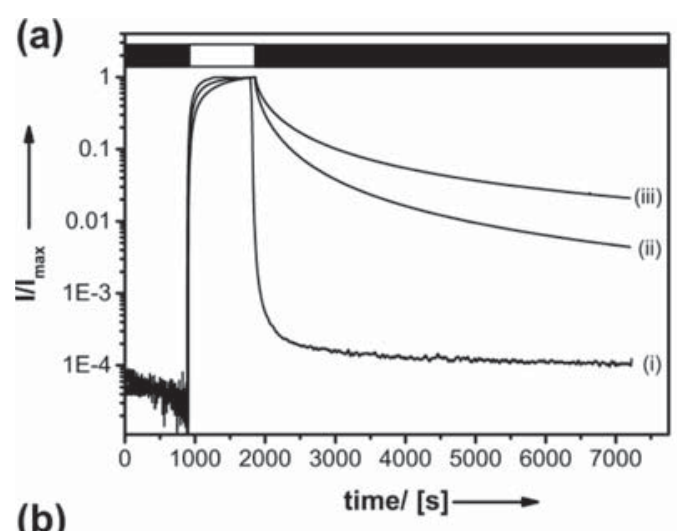

(b)

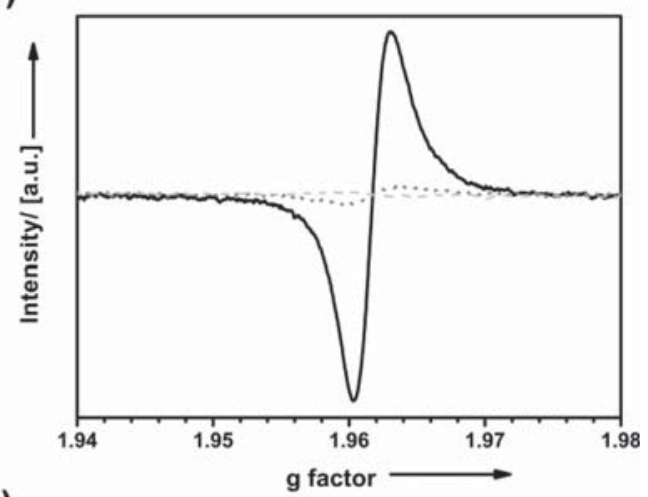

(c)

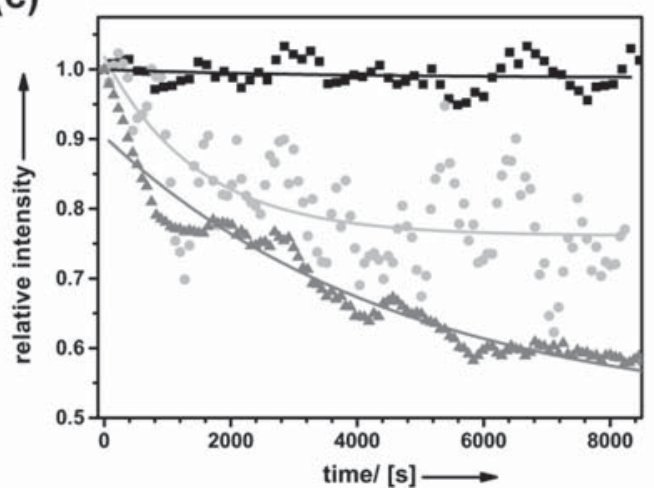

Figure 5. (a) Photodetector measurements: (i) bulk $\mathrm{ZnO}$ as a reference; (ii) yolk-shell particles; (iii) hollow particles. The illumination period is indicated by the white bar at the top of the graph. (b) EPR spectra of bulk $\mathrm{ZnO}$ during (dotted, gray curve) and after (dashed gray curve) UV radiation, in comparison to hollow $\mathrm{ZnO}$ particle ( $1 \mathrm{~h}$ after radiation; black curve). (c) Decay curves of the signal at $g=1.96$ for the compact spheres (dark gray triangles), the yolk-shell spheres (light gray dots), and the hollow spheres (black squares).

transmission decreases with UV exposure by approximately $4 \%$. This is a clear indication of free carrier absorption induced in the UV irradiated samples. Forty minutes after the UV exposure, the transmissions roughly recover to $50 \%$ of the value in the nonilluminated case.

Our findings can be summarized as follows. Like for conventional semiconductor materials, irradiation with light of sufficient energy leads to the formation of PGCCs. However, it seems that in the case of the studied nanospheres, the PGCCs are rapidly transferred into a metastable state, in which electrons and holes are separated effectively but electrons are still partially mobile. We suggest that the electric field resulting from the polar character of the nanocrystals in the $\mathrm{ZnO}$ shell (see Figure $3 \mathrm{~g}$ ) might be responsible for the mentioned process. ${ }^{94}$ Electrons and holes quickly diffuse to different interfaces of the hollow particles where they are trapped; for example, electrons move to the external interface and holes to the internal interface. Once the electrons have reached the outer surface, the probability to recombine with holes is significantly reduced since the holes are located in a spatially different domain. A persistent EPR signal, as observed in the hollow spheres, can be expected. Moreover, this model also explains the increased conductivity over a prolonged period of time, as the spatially separated PGCCs would still maintain their mobility. Additional support for this model comes from low temperature photoluminescence (PL) measurements. PL spectra normalized to the basal plane stacking fault (e, A) transition at $3.314 \mathrm{eV}$ are shown for the hollow $\mathrm{ZnO}$ spheres and compact spheres in Figure $4 \mathrm{~b}$. The PL spectrum of the compact spheres reveals a comparable intensity of the donor bound excitons $\left(\mathrm{D}^{0} \mathrm{X}\right)$ and surface bound excitons (SX) emission lines. ${ }^{75}$ However, in the case of the hollow $\mathrm{ZnO}$ spheres, the signal associated with the surface bound exciton is strongly increased. This indicates that charge carriers located near the surfaces are of significant importance for this particular morphology.

Additional information about strain and crystalline quality of the nanospheres can be obtained by the Raman spectrum in Figure 4a. Since the $\mathrm{E}_{2}$ modes are nonpolar modes of the $\mathrm{Zn}$ and $O$ sublattices and therefore unaffected by carrier concentration, they are often used to measure strain provided that the temperature is constant. ${ }^{76}$ The large hydrostatic pressure coefficient of the $\mathrm{E}_{2}^{\text {high }}$ mode $\left(5.04 \mathrm{~cm}^{-1} / \mathrm{GPa}\right)$ qualifies this particular mode as a sensitive sensor for strain in the nanospheres. ${ }^{55}$ The negligible variation in the position of the $\mathrm{E}_{2}^{\text {high }}$ mode with respect to its position in unstrained bulk $\mathrm{ZnO}^{20}$ demonstrates the absence of strain in the nanospheres. The excellent crystalline quality of the nanospheres is furthermore demonstrated by the very narrow line width of the $\mathrm{E}_{2}^{\text {low }}$ Raman mode. In contrast to the $\mathrm{E}_{2}^{\text {high }}$ mode, which is asymmetrically broadened due to the anharmonic decay into zone edge phonons, ${ }^{77}$ the $\mathrm{E}_{2}^{\text {low }}$ mode has a very small line width of $1.8 \mathrm{~cm}^{-1}$, which is comparable with state of the art $\mathrm{ZnO}$ substrates. The Raman data provides convincing arguments for the conclusion that no other structural imperfections than the special, polar hollow sphere morphology are responsible for the observed effects.

Thermal Conductivity. In addition to the structural and photophysical properties, we have investigated the thermal conductivity $(\kappa)$ of the hollow nanospheres using photo luminescence thermometry (see Experimental Section). Figure 7 displays the temperature rise, that is, energy shift of the band edge luminescence (Supporting Information, Figure S 10), in the hollow nanospheres as a function of the incident laser power.

Measurements of a bulk $\mathrm{ZnO}$ sample purchased from Tokyo Denpa are shown in the same figure for relative comparison. The thermal conductivity of the samples is obtained using the semi infinite medium approximation as described in the Experimental Section. For the bulk sample, we obtain $\kappa_{\text {bulk }}=$ $38 \pm 2 \mathrm{~W} / \mathrm{mK}$, in excellent agreement with the values reported in ref 78. In case of the hollow nanospheres, a value of $\kappa_{\text {eff }}=$ $0.13 \pm 0.01 \mathrm{~W} / \mathrm{mK}$ is determined. It should be noted that $\kappa_{\text {eff }}$ is only an effective value since it includes the contributions from 

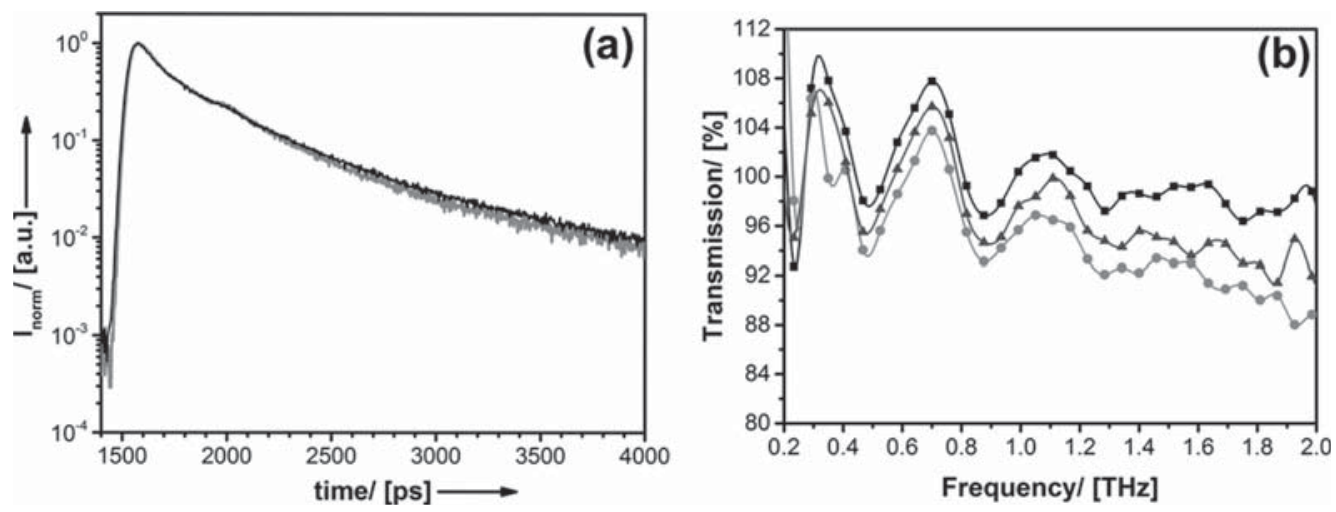

Figure 6. (a) TRPL data (e, A transition) for the compact $\mathrm{ZnO}$ spheres (gray graph) compared with the hollow $\mathrm{ZnO}$ spheres (black graph). (b) Transmission spectrum of yolk-shell spheres in the range from 0.2 to $2.0 \mathrm{THz}$ without UV exposure (black squares), with UV irradiation (light gray dots), and $40 \mathrm{~min}$ after UV exposure (dark gray triangles).

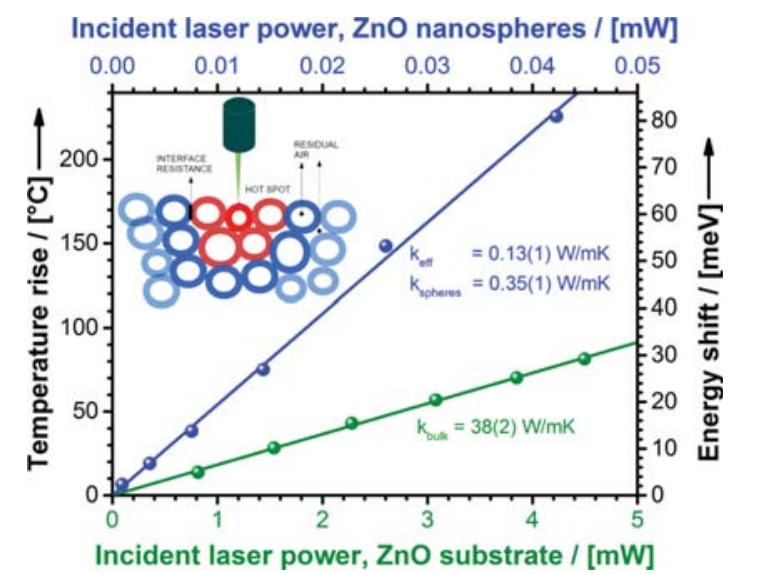

Figure 7. Temperature rise and energy shift of the band edge luminescence as a function of incident laser power. Bottom scale, laser power for a bulk $\mathrm{ZnO}$ substrate; top scale, incident power for hollow nanospheres. Inset: schematic illustration of laser induced heating and heat propagation in the hollow nanospheres.

the residual air $\left(\kappa_{\text {air }}=0.024 \mathrm{~W} / \mathrm{mK}\right)$ contained in the hollow spheres and between neighboring spheres. Considering that the filling fraction of $\mathrm{ZnO}$ with respect to air in the hollow spheres is around $1 / 3$ (based on a statistical evaluation of the scanning electron images), we can decouple the contribution of the $\mathrm{ZnO}$ spheres from that of the contained air and obtain a value of $\kappa_{\text {spheres }}=0.35 \pm 0.01 \mathrm{~W} / \mathrm{mK}$. Whereas the later value is representative for the thermal conductivity of the $\mathrm{ZnO}$ material, the former value of $k_{\text {eff }}=0.13 \pm 0.01 \mathrm{~W} / \mathrm{mK}$ is more realistic if the thermal properties of large scale clusters of hollow spheres are required. This large reduction in $\kappa$ in the nanospheres compared with the bulk value for $\mathrm{ZnO}$ arises mostly from the high thermal resistance between the nanospheres and the influence of surface phonon scattering within a single sphere. Considering that the thermoelectric figure of merit (ZT) scales inversely with $\kappa$, the low thermal conductivity together with the pronounced photoconductivity is extremely promising for applications in thermoelectric energy conversion.

\section{CONCLUSION}

Spherical zinc oxide nanomaterials were synthesized by gas phase synthesis in the size regime $>50 \mathrm{~nm}$. By changing the kinetics of crystallization, we were able to synthesize porous, dense, hollow and yolk/shell spheres. The generation of the hollow spheres and the shells in the yolk/shell structures could be attributed to the Kirkendall effect. UV photodetectors were built from the spheres, which showed exceptionally enhanced lifetime for the photogenerated charge carriers. The source of the enhanced lifetime was investigated using EPR, photo luminescence, TEM, Raman, and terahertz spectroscopy. It was found that the polar surfaces of $\mathrm{ZnO}$ align perpendicular to the spheres' core, which leads to a charge separation of the two types of charge carriers between the inner surface and the outer surface. It is suggested that this separation leads to a stabilization of the charge carriers along the polar planes, which constitutes an important advantage for applications in photocatalysis and photovoltaics, as well as enhanced catalytic performance of $\mathrm{ZnO}$ in water splitting and methanol synthesis. Furthermore, it could be demonstrated that the thermal conductivity of the samples comprising hollow $\mathrm{ZnO}$ spheres is extraordinary low. Both factors together, sufficient electric conductivity and high thermal resistance, are prerequisites for the construction of efficient thermoelectric devices. A thermo electric material based on a cheap, abundant, and environ mentally stable material such as these $\mathrm{ZnO}$ nanostructures, is a most promising goal, which constitutes a strong motivation for continued and future research beyond the scope of the current work.

\section{ASSOCIATED CONTENT}

\section{Supporting Information}

Aerosol setup and formation mechanism of the large, amorphous $\left[\mathrm{HOZnO}\left(\mathrm{HOCH}_{2} \mathrm{CH}_{2} \mathrm{OMe}\right)\right]_{n}$ particles, addi tional characterization of the amorphous spheres, trans formation of the amorphous particles into $\mathrm{ZnO}$ at different temperatures, characterization of the mesoporous $\mathrm{ZnO}$ particles obtained at different temperatures, additional analytical data for the sample prepared at $T=400{ }^{\circ} \mathrm{C}$, additional analytical data for the sample prepared at $T=600{ }^{\circ} \mathrm{C}$, PXRD patterns for hollow $\mathrm{ZnO}$ particles prepared at different temperatures, additional analytical data for the hollow sphere sample, photodetector measurements for the porous $\mathrm{ZnO}$ particles, representative photoluminescence emission from the $\mathrm{ZnO}$ hollow spheres compared with a bulk $\mathrm{ZnO}$ sample, and luminescence maximum shifts to larger wavelengths as the absorbed power is increased. This material is available free of charge via the Internet at http://pubs.acs.org. 


\section{AUTHOR INFORMATION}

\section{Corresponding Author}

*E mail: sebastian.polarz@uni konstanz.de. Fax: (+49) 7531 884406.

\section{Author Contributions}

The manuscript was written through contributions of all authors. All authors have given approval to the final version of the manuscript.

Notes

The authors declare no competing financial interest.

\section{ACKNOWLEDGMENTS}

SP acknowledges the German Research Foundation (Grant PO 780/8 1) and the Carl Zeiss Foundation (project Refine) for funding. ICN2 acknowledges financial support from the Spanish MICINN projects TAPHOR (Grant MAT2012 31392), nanoTHERM (Grant No. CSD2010 0044), and the FP7 projects NANOTEG ENIAC (Grant No. 270789 2), NANOTHERM (Grant No. 318117), and MERGING (Grant No. 309150). M.R.W. gratefully acknowledges the Marie Curie Fellowship (IEF) HeatProNano (Grant No. 628197). L.Q. acknowledges support through the China Scholarship Coucil.

\section{REFERENCES}

(1) Jain, S. C.; Willander, M.; Narayan, J.; Van Overstraeten, R. J. Appl. Phys. 2000, 87, 965.

(2) Serpone, N.; Dondi, D.; Albini, A. Inorg. Chim. Act. 2007, 360, 794

(3) Barbe, C. J.; Arendse, F.; Comte, P.; Jirousek, M.; Lenzmann, F.; Shklover, V.; Gratzel, M. J. Am. Ceram. Soc. 1997, 80, 3157.

(4) Law, M.; Greene, L. E.; Johnson, J. C.; Saykally, R.; Yang, P. D. Nat. Mater. 2005, 4, 455.

(5) Kudo, A.; Miseki, Y. Chem. Soc. Rev. 2009, 38, 253

(6) Zou, Z. G.; Ye, J. H.; Sayama, K.; Arakawa, H. Nature 2001, 414, 625.

(7) Martinez Huitle, C. A.; Brillas, E. Appl. Catal., B 2009, 87, 105.

(8) Greenham, N. C.; Peng, X.; Alivisatos, A. P. Phys. Rev. B 1996, 54, 17628.

(9) Van de Walle, C. G. Physica B 2001, 308-310, 899.

(10) Kröger, F. A. The Chemistry of Imperfect Crystals; North Holland Pub. Co.: Amsterdam, 1974.

(11) Janotti, A.; Van de Walle, C. G. Phys. Rev. B 2007, 76, No. 165202.

(12) Zhang, S. B.; Wei, S. H.; Zunger, A. Phys. Rev. B 2001, 63, No. 075205

(13) Wang, Z. L. J. Phys.: Condens. Matter 2004, 16, R829.

(14) Schmidt Mende, L.; MacManus Driscoll, J. L. Mater. Today 2007, 10, 40 .

(15) Zhang, J.; Sun, L. D.; Yin, J. L.; Su, H. L.; Liao, C. S.; Yan, C. H. Chem. Mater. 2002, 14, 4172.

(16) Khomenkova, L.; Fernandez, P.; Piqueras, J. Cryst. Growth Des. 2007, 7, 836.

(17) Seelig, E. W.; Tang, B.; Yamilov, A.; Cao, H.; Chang, R. P. H. Mater. Chem. Phys. 2003, 80, 257.

(18) Mo, M.; Yu, J. C.; Zhang, L. Z.; Li, S. K. A. Adv. Mater. 2005, 17, 756.

(19) Kuo, C. L.; Kuo, T. J.; Huang, M. H. J. Phys. Chem. B 2005, 109, 20115.

(20) Wang, D. B.; Song, C. X.; Hue, Z. S. Cryst. Res. Technol. 2008, 43,55 .

(21) Jiang, H.; Hu, J. Q.; Gu, F.; Li, C. Z. J. Phys. Chem. C 2008, 112, 12138 .

(22) Cheng, H. M.; Hsu, H. C.; Chen, S. L.; Wu, W. T.; Kao, C. C.; Lin, L. J.; Hsieh, W. F. J. Cryst. Growth 2005, 277, 192.
(23) Zhang, J.; Yang, Y. D.; Xu, B. L.; Jiang, F. H.; Li, J. P. J. Cryst. Growth 2005, 280, 509 .

(24) Wang, L. L.; Lou, Z.; Fei, T.; Zhang, T. J. Mater. Chem. 2011, $21,19331$.

(25) Liu, J.; Qiao, S. Z.; Chen, J. S.; Lou, X. W.; Xing, X. R.; Lu, G. Q. Chem. Commun. 2011, 47, 12578.

(26) Yin, Y.; Rioux, R. M.; Erdonmez, C. K.; Hughes, S.; Somorjai, G. A.; Alivisatos, A. P. Science 2004, 304, 711.

(27) Cho, W.; Lee, Y. H.; Lee, H. J.; Oh, M. Adv. Mater. 2011, 23, 1720.

(28) Sun, Y. G.; Wiley, B.; Li, Z. Y.; Xia, Y. N. J. Am. Chem. Soc. 2004, 126, 9399.

(29) Gao, J. H.; Liang, G. L.; Zhang, B.; Kuang, Y.; Zhang, X. X.; Xu, B. J. Am. Chem. Soc. 2007, 129, 1428 .

(30) Gao, J. H.; Liang, G. L.; Cheung, J. S.; Pan, Y.; Kuang, Y.; Zhao, F.; Zhang, B.; Zhang, X. X.; Wu, E. X.; Xu, B. J. Am. Chem. Soc. 2008, 130, 11828.

(31) Chen, D.; Li, L. L.; Tang, F. Q.; Qi, S. O. Adv. Mater. 2009, 21, 3804.

(32) Chen, J. S.; Li, C. M.; Zhou, W. W.; Yan, Q. Y.; Archer, L. A.; Lou, X. W. Nanoscale 2009, 1, 280.

(33) Pandey, A. D.; Guttel, R.; Leoni, M.; Schuth, F.; Weidenthaler, C. J. Phys. Chem. C 2010, 114, 19386.

(34) Tan, L. F.; Chen, D.; Liu, H. Y.; Tang, F. Q. Adv. Mater. 2010, 22, 4885.

(35) Krumm, M.; Pueyo, C. L.; Polarz, S. Chem. Mater. 2010, 22, 5129.

(36) Krumm, M.; Pawlitzek, F.; Weickert, J.; Schmidt Mende, L.; Polarz, S. ACS Appl. Mater. Interfaces 2012, 4, 6522.

(37) Dilger, S.; Lizandara Pueyo, C.; Krumm, M.; Polarz, S. Adv. Mater. 2012, 24, 543.

(38) Reparaz, J. S.; Chavez Angel, E.; Wagner, M. R.; Graczykowski, B.; Gomis Bresco, J.; Alzina, F.; Sotomayor Torres, C. M. Rev. Sci. Instrum. 2014, 85, No. 034901.

(39) McDonald, F. A.; Wetsel, G. C., Jr. Theory of photoacoustic and photothermal effects in condensed matter. In Physical Acoustics; Mason, W. P., Thurston, R. N., Eds.; Academic Press: San Diego, CA, 1988; Vol. 18, p 167.

(40) Nonnenmacher, M.; Wickramasinghe, H. K. Appl. Phys. Lett. 1992, 61, 168.

(41) Cobet, M.; Cobet, C.; Wagner, M. R.; Esser, N.; Thomsen, C.; Hoffmann, A. Appl. Phys. Lett. 2010, 96, 3.

(42) Wang, L. J.; Giles, N. C. J. Appl. Phys. 2003, 94, 973.

(43) Hahn, H. Nanostruct. Mater. 1997, 9, 3.

(44) Kruis, F. E.; Fissan, H.; Peled, A. J. Aerosol Sci. 1998, 29, 511.

(45) Boissiere, C.; Grosso, D.; Chaumonnot, A.; Nicole, L.; Sanchez, C. Adv. Mater. 2011, 23, 599.

(46) Polarz, S.; Roy, A.; Lehmann, M.; Driess, M.; Kruis, F. E.; Hoffmann, A.; Zimmer, P. Adv. Funct. Mater. 2007, 17, 1385.

(47) Polarz, S.; Strunk, J.; Ischenko, V.; Van den Berg, M.; Hinrichsen, O.; Muhler, M.; Driess, M. Angew. Chem. 2006, 118, 3031.

(48) Ischenko, V.; Polarz, S.; Grote, D.; Stavarache, V.; Fink, K.; Driess, M. Adv. Funct. Mater. 2005, 15, 1945.

(49) Polarz, S.; Orlov, A. V.; Schueth, F.; Lu, A. H. Chem.-Eur. J. 2007, 13, 592

(50) Polarz, S.; Roy, A.; Merz, M.; Halm, S.; Schroder, D.; Schneider, L.; Bacher, G.; Kruis, F. E.; Driess, M. Small 2005, 1, 540.

(51) Polarz, S.; Neues, F.; Van den Berg, M.; Grünert, W.; Khodeir, L. J. Am. Chem. Soc. 2005, 127, 12028.

(52) Lizandara Pueyo, C.; Siroky, S.; Wagner, M. R.; Hoffmann, A.; Reparaz, J. S.; Lehmann, M.; Polarz, S. Adv. Funct. Mater. 2011, 21, 295.

(53) Lizandara Pueyo, C.; Dilger, S.; Wagner, M. R.; Gerigk, M.; Hoffmann, A.; Polarz, S. CrystEngComm 2014, 16, 1525.

(54) Smigelskas, A. D.; Kirkendall, E. O. Trans. Am. Inst. Mining Metall. Eng. 1947, 171, 130.

(55) Reparaz, J. S.; Muniz, L. R.; Wagner, M. R.; Goñi, A. R.; Alonso, M. I.; Hoffmann, A.; Meyer, B. K. Appl. Phys. Lett. 2010, 96, No. 231906 
(56) Cusco, R.; Alarcon Llado, E.; Ibanez, J.; Artus, L.; Jimenez, J.; Wang, B. G.; Callahan, M. J. Phys. Rev. B 2007, 75, 11.

(57) Takeuchi, T.; Wetzel, C.; Yamaguchi, S.; Sakai, H.; Amano, H.; Akasaki, I.; Kaneko, Y.; Nakagawa, S.; Yamaoka, Y.; Yamada, N. Appl. Phys. Lett. 1998, 73, 1691.

(58) Wang, W.; Qi, J.; Wang, Q.; Huang, Y.; Liao, Q.; Zhang, Y. Nanoscale 2013, 5, 5981.

(59) Lupan, O.; Chow, L.; Chai, G. Sens. Actuators, B 2009, 141, 511.

(60) Fang, X.; Bando, Y.; Liao, M.; Gautam, U. K.; Zhi, C.; Dierre, B.; Liu, B.; Zhai, T.; Sekiguchi, T.; Koide, Y.; Golberg, D. Adv. Mater. 2009, 21, 2034.

(61) Drouilly, C.; Krafft, J. M.; Averseng, F.; Casale, S.; Bazer Bachi, D.; Chizallet, C.; Lecocq, V.; Vezin, H.; Lauron Pernot, H.; Costentin, G. J. Phys. Chem. C 2012, 116, 21297.

(62) Sancier, K. M. Surf. Sci. 1970, 21, 1.

(63) Morazzoni, F.; Scotti, R.; Dinola, P.; Milani, C.; Narducci, D. J. Chem. Soc., Faraday Trans. 1992, 88, 1691.

(64) Studenikin, S. A.; Cocivera, M. J. Appl. Phys. 2002, 91, 5060.

(65) Studenikin, S. A.; Golego, N.; Cocivera, M. J. Appl. Phys. 1998, $83,2104$.

(66) Studenikin, S. A.; Golego, N.; Cocivera, M. J. Appl. Phys. 1998, 84,5001 .

(67) Studenikin, S. A.; Golego, N.; Cocivera, M. J. Appl. Phys. 1998, $84,2287$.

(68) Studenikin, S. A.; Golego, N.; Cocivera, M. J. Appl. Phys. 2000, $87,2413$.

(69) Rauch, C.; Gehlhoff, W.; Wagner, M. R.; Malguth, E.; Callsen, G.; Kirste, R.; Salameh, B.; Hoffmann, A.; Polarz, S.; Aksu, Y.; Driess, M. J. Appl. Phys. 2010, 107, 5.

(70) Baxter, J. B.; Schmuttenmaer, C. A. J. Phys. Chem. B 2006, 110, 25229.

(71) Lloyd Hughes, J.; Jeon, T. I. J. Infrared, Millimeter, Terahertz Waves 2012, 33, 871.

(72) Balci, S.; Baughman, W.; Wilbert, D. S.; Shen, G.; Kung, P.; Kim, S. M. Solid State Electron. 2012, 78, 68.

(73) Gebs, R.; Klatt, G.; Janke, C.; Dekorsy, T.; Bartels, A. Opt. Express 2010, 18, 5974.

(74) Polland, H. J.; Schultheis, L.; Kuhl, J.; Gobel, E. O.; Tu, C. W. Phys. Rev. Lett. 1985, 55, 2610.

(75) Wagner, M. R.; Callsen, G.; Reparaz, J. S.; Schulze, J. H.; Kirste, R.; Cobet, M.; Ostapenko, I. A.; Rodt, S.; Nenstiel, C.; Kaiser, M.; Hoffmann, A.; Rodina, A. V.; Phillips, M. R.; Lautenschlager, S.; Eisermann, S.; Meyer, B. K. Phys. Rev. B 2011, 84, No. 035313.

(76) Wagner, M. R.; Bartel, T. P.; Kirste, R.; Hoffmann, A.; Sann, J.; Lautenschläger, S.; Meyer, B. K.; Kisielowski, C. Phys. Rev. B 2009, 79, No. 035307.

(77) Serrano, J.; Manjon, F. J.; Romero, A. H.; Widulle, F.; Lauck, R.; Cardona, M. Phys. Rev. Lett. 2003, 90, No. 055510.

(78) Olorunyolemi, T.; Birnboim, A.; Carmel, Y.; Wilson, O. C.; Lloyd, I. K.; Smith, S.; Campbell, R. J. Am. Ceram. Soc. 2002, 85, 1249. 